Wang, Z. and Li, P. (2020) Visco-elasto-plastic constitutive model of adhesives under uniaxial compression in a range of strain rates. Journal of Applied Polymer Science, 137(33), 48962.

There may be differences between this version and the published version. You are advised to consult the publisher's version if you wish to cite from it.

This is the peer reviewed version of the following article:

Wang, Z. and Li, P. (2020) Visco-elasto-plastic constitutive model of adhesives under uniaxial compression in a range of strain rates. Journal of Applied Polymer Science, 137(33), 48962. (doi: 10.1002/app.48962)

This article may be used for non-commercial purposes in accordance with Wiley Terms and Conditions for Self-Archiving.

http://eprints.gla.ac.uk/208344/

Deposited on: 24 January 2020

Enlighten - Research publications by members of the University of Glasgow http://eprints.gla.ac.uk/ 


\section{A visco-elasto-plastic constitutive model of adhesives under uniaxial compression in a range of strain rates}

Zhiyong Wang a , Peifeng $\mathrm{Li}^{\mathrm{b}, *}$

${ }^{a}$ School of Mechatronical Engineering, Beijing Institute of Technology, Beijing, China

${ }^{b}$ James Watt School of Engineering, University of Glasgow, Glasgow, UK

*Corresponding author's email: peifeng.li@glasgow.ac.uk (P. Li); Tel: +44 1413302703. 


\section{Abstract}

\section{Introduction}

The room temperature curable polymer is one of the most preferably used adhesives that offers the superior interfacial bonding strength in hybrid structures such as fibre metal laminates ${ }^{[1-4]}$. Due to the excellent peel resistance and impact strength, these adhesive layers can be applied to structures such as in automobiles and wind turbines (e.g., to join composite blades to metal hubs). The dynamic performance of the structure can play a crucial role ${ }^{[5,6]}$ in these applications. Determining the high strain rate response of such structural adhesives requires not only the reliable experimental characterisation but also the quantitative constitutive prediction.

A number of experimental studies have been performed in the past decades on the mechanical behaviour of amorphous polymers ${ }^{[2,7-12]}$. It was found that the deformation is significantly affected by temperature and strain rate ${ }^{[13-15]}$. A bilinear relation was observed between the stress and the logarithm of strain rate for a variety of polymers ${ }^{[10,13-15]}$. The viscous nature of polymers or elastomers causes the apparent rate sensitivity (e.g., in modulus and yield stress) even in the low loading rate range ${ }^{[16-20]}$. The reliable measurement of dynamic behaviour of polymers offers the physical insight in the development of a constitutive model. However, it is still 
challenging to experimentally measure the high rate response of a soft polymeric material ${ }^{[18]}$.

Various constitutive models have been developed to represent the mechanical behaviour of polymeric materials on the basis of experimental observations ${ }^{[16,21-28]}$. Phenomenological models using empirical equations are widely accepted as they are practically simple; and they can well describe the nonlinear deformation behaviour ${ }^{[18 \text {, }}$ 23, 29]. An elasto-viscoplastic constitutive formula coupled with micromechanics and statistical thermodynamics has been proposed to simulate the response of toughened polymeric materials, such as toughened polycarbonate in both microscopic and macroscopic scales ${ }^{[30,31]}$. The nonlinear viscoelastic behaviour of epoxy-based adhesives can be quantified by rheological ${ }^{[25]}$ or time-hardening ${ }^{[32]}$ models. A constitutive model based on statistical mechanics was established for elastomers, incorporating the interacting macromolecular networks ${ }^{[16,33]}$. Many parameters are often required to best correlate the model with experimental data. To reduce the number of material parameters, a visco-hyperelastic constitutive model using a rate dependent relaxation time scheme was developed for rubber-like materials under large deformation ${ }^{[34]}$. Nonetheless, the model is only applicable to a limited range of loading rates. A constitutive model should consider the strain rate sensitivity to enable its wide applications.

The aim of the study reported here is to determine a constitutive model to quantify the strain rate sensitive compressive behaviour of a two-component structural 
adhesive based on the experimental measurements. Uniaxial compression experiments were performed to measure the mechanical response at the strain rates ranging from 0.001 to $2600 \mathrm{~s}^{-1}$. The strain rate sensitivity using empirical equations was proposed into a visco-elasto-plastic constitutive model for the adhesive.

\section{Experimental procedure}

Hysol EA9309.3NA (Henkel AG, Germany) is a toughened two-component epoxy adhesive that offers high shear and peel strength to metals (e.g., aluminium). Cylindrical Hysol adhesive specimens for uniaxial compression experiments were prepared with the nominal diameter $d=10 \mathrm{~mm}$ and length $l=15,10,6$ and $4 \mathrm{~mm}$. The respective aspect ratio was $l / d=1.5,1.0,0.6$ and 0.4 . The quasi-static uniaxial compression experiments were performed on the high aspect ratio specimens $(l / d=$ 1.5 and 1.0) in an INSTRON 5569 (INSTRON, MA, USA) electromechanical universal testing machine (respective strain rate $\dot{\varepsilon}=0.001$ and $0.1 \mathrm{~s}^{-1}$ ). The real-time deformation process was captured using a JAI (JAI Ltd., Japan) BM-500 GE high resolution camera.

The low aspect ratio specimens $(l / d=0.6$ and 0.4$)$ were subjected to dynamic compression in a split-Hopkinson pressure bar (SHPB) system. The SHPB system consisted of the striker (length $800 \mathrm{~mm})$, input $(2000 \mathrm{~mm})$ and output $(2000 \mathrm{~mm})$ bars. All the bars of $20 \mathrm{~mm}$ in diameter were made of aluminium alloy AA7075. The $800 \mathrm{~mm}$ long striker produced a loading duration sufficient for a large deformation in 
the adhesive specimen. Three dynamic strain rates $\dot{\varepsilon}=1000,2000$ and $2600 \mathrm{~s}^{-1}$ were achieved in SHPB testing. The specimen ends in all the quasi-static and dynamic tests were lubricated with Castrol LMX grease to minimise the interfacial friction.

The incident, reflected and transmitted strain wave signals were measured with the strain gauges mounted in the middle of the bars and then subjected to noise reduction by fast Fourier transformation (Fig. 1(a)). The wave signals were reconstructed at the bar-specimen interfaces, from which the resisting force and deformation rate of a specimen were calculated. The difference between the axial forces at the input $\left(F_{\text {in }}\right)$ and output $\left(F_{\text {out }}\right)$ ends of the specimen was normalised by their average $\left(\left(F_{\text {in }}+F_{\text {out }}\right) / 2\right)$,

$$
\eta=2\left|\frac{F_{\text {in }}-F_{\text {out }}}{F_{\text {in }}+F_{\text {out }}}\right|
$$

where $\eta$ is the parameter to characterise the force equilibrium. The parameter $\eta$ is less than $6 \%$ for the SHPB load that can be indicative of the force equilibrium (refer to Fig. 2). Force equilibrium at the specimen ends established at the early strain stage suggests the insignificant inertial effect on the measured mechanical response. Thus, the stress and strain histories were finally calculated in the specimen (Fig. 1(b)). The strain linearly increases with time in the majority of the SHPB load. The dynamic strain rate is defined as the slope of the linear portion. The SHPB system, analysis of recorded wave signals and verification of stress uniformity were detailed in the authors' previous studies on several different materials ${ }^{[18,35-37]}$. 


\section{Results and discussion}

\subsection{Stress-strain response}

Fig. 3 shows the typical nominal stress-strain response of Hysol adhesives under uniaxial compression at the strain rates ranging from 0.001 to $2600 \mathrm{~s}^{-1}$. At least four specimens were tested under each strain rate condition. The tests at all the five strain rates can be repeated with a deviation of $<5 \%$, indicative of consistent specimen geometry and testing conditions between specimens. Note that the maximum strain achieved at the strain rate $1000 \mathrm{~s}^{-1}$ is only approximately 0.3 . This is caused by the limited width of the incident pulse (i.e., the loading duration) in the SHPB test, which is determined by the striker bar length $(800 \mathrm{~mm})$ and the wave velocity in the AA7075 bar. The bulk stress-strain curves have the similar characteristics for all the different rates. The elastic deformation is initially approximately linear, and then nonlinear. The first peak value in the curve is defined as the yield strength $\sigma_{\mathrm{y}}$. Beyond the yield point, the flow stress first decreases and then increases with the strain, implying the strain softening and hardening behaviour that dominate at different strain stages.

Fig. 4 illustrates the deformation process of an adhesive specimen subjected to quasi-static compression at the strain rate $\dot{\varepsilon}=0.001 \mathrm{~s}^{-1}$. Slight barrelling arises at the large strains due to the interfacial friction at the specimen ends. The specimen volume ( $V$ ) was calculated from the recorded 2D image with the assumption of axisymmetric 
longitudinal deformation, and then normalised by the original volume $\left(V_{0}\right)$. The procedure details can be found in the previous work ${ }^{[18]}$. As shown in Fig. 4, the specimen volume change is less than $1 \%$, implying that the adhesive material is incompressible.

\subsection{Effect of strain rates}

The initial elastic modulus increases with the strain rate (Fig. 5). Note that the initial modulus at the dynamic rates was calculated from the upper linear portion (stress $>50 \mathrm{MPa}$ ) of the elastic deformation in the curves, where the force equilibrium parameter decreased to $\eta<6.0 \%$ (Figs. 1(b) and 2). The strain rate sensitivity of the modulus is caused by the viscosity of the adhesive. The constitutive elastic model for a wide range of loading rates should therefore consider the effect of strain rates. The yield strength also increases with the strain rate (Fig. 6). The previous research suggests that the impact of strain rate on the yield stress is due to the secondary molecular process ${ }^{[38-41]}$. Increasing the deformation rate makes the polymer stiffer, thus resulting in the lower mobility of the molecule.

The true flow stress was calculated from the measured nominal stress-strain data with the assumption of no volume change during the plastic deformation (refer to Fig. 4). The true stress was then normalised by the true yield strength at the given strain rate. Fig. 7 illustrates the variation of the normalised true flow stress with the logarithm of strain rates at seven selected true plastic strains $\varepsilon_{\mathrm{pl}}=0.1-0.7$. The strain 
rate sensitivity represented by the slope of each curve in Fig. 7 is not constant, but increases with the strain rate, i.e., the slope being from negative to positive. At a specific strain rate, the true flow stress first decreases with the plastic strain when $\varepsilon_{\mathrm{pl}}<$ 0.3 , and subsequently increases with the strain when $\varepsilon_{\mathrm{pl}}>0.3$ (Fig. 7). This further suggests that strain softening dominates at low strains whilst strain hardening at high strains. Therefore, the constitutive plastic model should incorporate strain softening, strain hardening and strain rate effects.

\subsection{A visco-elasto-plastic constitutive model}

Based on the experimental observations, a visco-elasto-plastic constitutive model was proposed to quantify the strain rate dependent compressive behaviour of the Hysol adhesive. The model describes the initial viscoelastic behaviour, the yielding point, the viscoplastic behaviour combining strain softening and hardening, and the strain rate effects on these phenomena.

\subsubsection{Viscoelastic behaviour}

The viscoelastic behaviour of Hysol adhesives can be characterised by a Maxwell model that contains an elastic spring and a viscous damper in series (Fig. 8). The stress $\sigma$ can be derived by integrating the following term over the time $t$ :

$$
\sigma(t)=\int_{0}^{t} E \dot{\varepsilon} \exp \left(-\frac{t-\theta}{\tau}\right) d \theta
$$

where $E$ is the elastic modulus, $\tau=\eta / E$ is the relaxation time and $\eta$ is the viscosity. Note that the average strain rate is used to describe the dynamic test (Fig. 1(b)). Thus, 
the strain rate can be considered to remain constant in both the quasi-static and dynamic experiments, and the time is calculated by $t=\varepsilon / \dot{\varepsilon}$. The stress $\sigma$ is then related to the strain by the integration of Eq. (2).

$$
\sigma=E \tau \dot{\varepsilon}\left[1-\exp \left(-\frac{\varepsilon}{\dot{\varepsilon} \tau}\right)\right]
$$

Eq. (3) is only suitable for the general Maxwell element, e.g., with a constant elastic modulus $E$ and a constant relaxation time $\tau$. However, in the viscoelastic response both the elastic modulus and relaxation time are influenced by the temperature and loading rate $[11,15,21,41]$. In the present study, the temperature dependency can be neglected because the mechanical tests were conducted at room temperature. Eq. (3) can be rewritten to represent the viscoelastic stress $\sigma_{\mathrm{ve}}$ :

$$
\sigma_{v e}=E(\dot{\varepsilon}) \tau(\dot{\varepsilon}) \dot{\varepsilon}\left[1-\exp \left(-\frac{\varepsilon}{\dot{\varepsilon} \tau(\dot{\varepsilon})}\right)\right]
$$

where $E(\dot{\varepsilon})$ and $\tau(\dot{\varepsilon})$ are the strain rate sensitive functions for the initial elastic modulus and the relaxation time, respectively.

A term $\left(1+s \ln \left(\dot{\varepsilon} / \dot{\varepsilon}_{0}\right)\right)$ similar to that in the Johnson-Cook model was introduced to quantitatively describe the strain rate effect on the initial elastic modulus while the temperature sensitivity was ignored ${ }^{[42]}$ :

$$
E(\dot{\varepsilon})=E_{0}\left(1+s \ln \left(\frac{\dot{\varepsilon}}{\dot{\varepsilon}_{0}}\right)\right)
$$

where $\dot{\varepsilon}_{0}=1.0$ is the reference strain rate, $E_{0}$ is the initial elastic modulus at the reference rate, and $s$ is the strain rate sensitivity parameter for the elastic modulus. 
The molecular relaxation can be restricted if the stimuli time scale decreases (i.e., the strain rate increases) or the temperature reduces. The logarithm of relaxation time can be linearly related to the strain rate ${ }^{[43]}$ :

$$
\tau(\dot{\varepsilon})=\tau_{0}\left(\frac{\dot{\varepsilon}}{\dot{\varepsilon}_{0}}\right)^{-\beta}
$$

where $\tau_{0}$ is a time related parameter and $\beta$ is a dimensionless constant, both of which can be determined experimentally. The viscoelastic stress $\sigma_{\mathrm{ve}}$ is thus determined as a function of both strain and strain rate.

$$
\sigma_{v e}=E_{0}\left(1+s \ln \left(\frac{\dot{\varepsilon}}{\dot{\varepsilon}_{0}}\right)\right) \tau_{0}\left(\frac{\dot{\varepsilon}}{\dot{\varepsilon}_{0}}\right)^{-\beta} \dot{\varepsilon}\left[1-\exp \left(-\frac{\varepsilon}{\dot{\varepsilon} \tau_{0}}\left(\frac{\dot{\varepsilon}}{\dot{\varepsilon}_{0}}\right)^{\beta}\right)\right]
$$

\subsubsection{Yield strength}

The yielding behaviour of solid amorphous polymers has been extensively reported in the literature ${ }^{[9-11,17,19,41]}$. The cooperative model becomes one of the established models to characterise the yielding behaviour, because it considers the strain rate and temperature superposition principle of the yield stress ${ }^{[44,45]}$. For temperatures below the glass transition, the cooperative model can be used to determine the compressive yield strength $\sigma_{\mathrm{y}}$ as a function of strain rate in the following form:

$$
\frac{\sigma_{\mathrm{y}}}{T_{0}}=\frac{\sigma_{\mathrm{y} 0}}{T_{0}}+\frac{2 k}{V} \sinh ^{-1}\left(\frac{\dot{\varepsilon}}{\dot{\varepsilon}_{\mathrm{y}}}\right)^{1 / n}
$$

where $T_{0}=298 \mathrm{~K}$ (i.e., $25{ }^{\circ} \mathrm{C}$ ) is the room temperature, $\sigma_{\mathrm{y} 0}$ is the internal stress, $k$ is the Boltzmann constant, $V$ is the arbitrary activation value, and $\dot{\varepsilon_{\mathrm{y}}}$ is the 
characteristic strain rate, and $n$ is the material constant. These parameters can be fitted from the experimental data.

\subsubsection{Viscoplastic behaviour}

To model the viscoplastic behaviour of Hysol adhesives, the strain softening and hardening responses are assumed to be independent. Beyond the yield point, the true flow stress is the sum of the yield strength, the increased stress due to hardening, and the decreased stress due to softening ${ }^{[46]}$ :

$$
\bar{\sigma}_{\mathrm{vp}}=1-\bar{\sigma}_{\mathrm{s}}+\bar{\sigma}_{\mathrm{h}}
$$

where $\bar{\sigma}_{\mathrm{vp}}=\sigma_{\mathrm{vp}} / \sigma_{\mathrm{y}}, \quad \bar{\sigma}_{\mathrm{s}}=\sigma_{\mathrm{s}} / \sigma_{\mathrm{y}}$ and $\bar{\sigma}_{\mathrm{h}}=\sigma_{\mathrm{h}} / \sigma_{\mathrm{y}}$ are the true viscoplastic stress $\sigma_{\mathrm{vp}}$, softening stress $\sigma_{\mathrm{s}}$ and hardening stress $\sigma_{\mathrm{h}}$ normalised by the yield strength $\sigma_{\mathrm{y}}$, respectively.

The internal shear resistance gives rise to the softening behaviour of polymeric materials. Based on the phenomenological description of internal stress evolution during strain softening ${ }^{[14]}$, the following function was proposed for the normalised softening stress at an effective plastic strain $\varepsilon_{\mathrm{p}}$ in the uniaxial compression test,

$$
\bar{\sigma}_{\mathrm{s}}=A\left(1-\exp \left(-k_{1} \varepsilon_{\mathrm{p}}\right)\right)
$$

where $A$ and $k_{1}$ are the softening parameters. The strain rate sensitivity of softening (Fig. 7) is quantified by integrating a factor $\left(1+D_{\mathrm{s}} \ln \left(\dot{\varepsilon} / \dot{\varepsilon}_{0}\right)\right)$ into the strain softening Eq. (10) ${ }^{[42]}$. The parameter $D_{\mathrm{s}}$ is not constant but varies with the strain rate in a power function, 


$$
D_{\mathrm{s}}=B\left(\frac{\dot{\varepsilon}}{\dot{\varepsilon}_{0}}\right)^{C}
$$

where $B$ and $C$ are the material constants for strain rate effects on softening. Thus the normalised softening stress $\bar{\sigma}_{\mathrm{s}}$ becomes.

$$
\bar{\sigma}_{\mathrm{s}}=A\left(1-\exp \left(-k_{1} \varepsilon_{\mathrm{p}}\right)\right)\left[1+B\left(\frac{\dot{\varepsilon}}{\dot{\varepsilon}_{0}}\right)^{C} \ln \left(\frac{\dot{\varepsilon}}{\dot{\varepsilon}_{0}}\right)\right]
$$

As the strain increases, hardening dominates the material behaviour. Physically, the hardening mechanisms for the viscoplastic material can be categorised into isotropic hardening and kinematic hardening. Isotropic hardening is related to the growth of the anelastic domain whilst kinematic hardening the presence of microstructural defects in the amorphous phase ${ }^{[47]}$. In terms of the viscoplasticity theory, isotropic hardening is incorporated into kinematic hardening to calculate the normalised hardening stress at a plastic strain $\varepsilon_{\mathrm{p}}$ :

$$
\bar{\sigma}_{\mathrm{h}}=D_{\mathrm{h}}\left(1-\exp \left(-k_{2} \varepsilon_{\mathrm{p}}\right)\right)
$$

where $k_{2}$ is a hardening constant, and the parameter $D_{\mathrm{h}}$ can be calculated according to the previous research:

$$
D_{\mathrm{h}}=\left(1+\varepsilon_{\mathrm{p}}\right)^{N}
$$

where $N$ is another hardening parameter. Therefore, the normalised hardening stress $\bar{\sigma}_{\mathrm{h}}$ becomes,

$$
\bar{\sigma}_{\mathrm{h}}=\left(1+\varepsilon_{\mathrm{p}}\right)^{N}\left(1-\exp \left(-k_{2} \varepsilon_{\mathrm{p}}\right)\right)
$$

Combining Eqs. $(9,12,15)$ leads to the normalised viscoplastic stress $\bar{\sigma}_{\mathrm{vp}}$. 


$$
\bar{\sigma}_{\mathrm{vp}}=1-A\left(1-\exp \left(-k_{1} \varepsilon_{\mathrm{p}}\right)\right)\left[1+B\left(\frac{\dot{\varepsilon}}{\dot{\varepsilon}_{0}}\right)^{C} \ln \left(\frac{\dot{\varepsilon}}{\dot{\varepsilon}_{0}}\right)\right]+\left(1+\varepsilon_{\mathrm{p}}\right)^{N}\left(1-\exp \left(-k_{2} \varepsilon_{\mathrm{p}}\right)\right)
$$

The material parameters $A, B, C, N, k_{1}$ and $k_{2}$ are dimensionless, which can be determined from the experimental results.

\subsection{Determination of material constants and model validation}

The regression analysis was performed on the measured stress-strain data at the four strain rates $\dot{\varepsilon}=0.001,0.1,1900$ and $2600 \mathrm{~s}^{-1}$ (Fig. 3(a, b, d and e)) to determine the material constants in the visco-elasto-plastic model (Table 1). Fig. 9 details the procedure to calculate these constants in sequence. First, the elastic modulus as measured from the initial linear portion of the stress-strain curves (Fig. 5) was analysed with Eq. (5) to fit the constants $E_{0}$ and $s$ (correlation coefficient $R^{2}=0.960$ ). The other two constants $\tau_{0}$ and $\beta$ for viscoelastic behaviour were determined by fitting the stress-strain data prior to the yield point with Eq. (7) $\left(R^{2}=0.997\right)$. The measured yield strength (Fig. 6) was then analysed to fit the material constants in Eq. (8) with $R^{2}=0.992$. Finally, the nominal stress and strain for the viscoplastic behaviour were converted to true stress and strain data. The parameters in Eq. (16) were fitted from the data $\left(R^{2}=0.981\right)$ and listed in Table 1 . The material constants affect the deformation behaviour of the adhesive in terms of the constitutive equations for the elastic and plastic deformations (Eqs. $(5,7,8,16))$. 
The measured stress-strain data at the strain rate $\dot{\varepsilon}=1000 \mathrm{~s}^{-1}$, excluded from the regression analysis, was used to validate the developed constitutive model and the fitted material constants. The predicted stress-strain curve is compared to the measured curve (Fig. 3(c)). Their good agreement suggests that the modified constitutive model is reliable to represent the compressive behaviour of Hysol adhesives in the range of strains and strain rates given in the present work.

The developed constitutive model may be applied to other structural adhesives if their stress-strain response is similar to the Hysol's visco-elasto-plastic behaviour. Future experiments at more strain rates especially the medium range $1.0-100 \mathrm{~s}^{-1}$ are required to generate a larger set of stress-strain data, in order to further validate the model and thus develop more complete constitutive equations. Note that mechanical properties of polymers are often dependent on temperature. If the adhesive is used in the temperature range other than that reported here, or the service temperature varies in the application, the temperature effect should be investigated and incorporated into the constitutive model.

\section{Conclusions}

The uniaxial compressive behaviour of a two-component structural adhesive was experimentally measured at the strain rates ranging from 0.001 to $2600 \mathrm{~s}^{-1}$ for the development of a visco-elasto-plastic constitutive model. The conclusions were drawn as follows. 
- The compression experiments reveal that the stress-strain behaviour is significantly influenced by the strain rate. Not only the yield strength but also the elastic modulus increases with the strain rate. Beyond the yield point, plastic deformation is dominated by strain softening at low strains and by strain hardening at high strains. The flow stress is sensitive to the strain rate; however, the rate sensitivity is not constant but increases with the strain rate.

- These strain rate effects on both the elastic and plastic deformations are quantified using specific empirical equations and incorporated into the proposed visco-elasto-plastic model. The modified constitutive model as experimentally validated can reliably characterise the compressive behaviour of the adhesive in the range of strains and strain rates.

Acknowledgements: The authors gratefully acknowledge the financial support of the National Natural Science Foundation of China (Grant No.: 11802028), the China Postdoctoral Science Foundation (2019M650502) and the Academic Research Fund (AcRF) Tier 1 by Ministry of Education in Singapore. ZW also thanks the Nanyang Technological University (NTU) Research Student Scholarship for his PhD study during which this work was carried out.

Keywords: Adhesives; Strain rates; Viscoelastic; Viscoplastic; Constitutive equation. 


\section{References}

[1] E. Degrandi-Contraires, A. Lopez, Y. Reyes, J. M. Asua, C. Creton, Macromol. Mater. Eng. 2013, 298, 612.

[2] L. Goglio, L. Peroni, M. Peroni, M. Rossetto, Int. J. Adhes. Adhes. 2008, 28, 329.

[3] A. Lopez, Y. Reyes, E. Degrandi-Contraires, E. Canetta, C. Creton, J. M. Asua, European Polymer Journal 2013, 49, 1541.

[4] R. Huang, P. Li, T. Liu, J. Xu, J. Appl. Polym. Sci. 2019, 136, 7.

[5] M. Cocconcelli, A. Spaggiari, J. Adhes. 2017, 93, 585.

[6] S. Sassi, M. Tarfaoui, H. Ben Yahia, Compos. Struct. 2018, 191, 168.

[7] B. H. Liu, J. Xu, Y. B. Li, Adv. Mater. Sci. Eng. 2014, 10.

[8] S. N. Raman, T. Ngo, J. Lu, P. Mendis, Mater. Des. 2013, 50, 124.

[9] K. H. Safari, J. Zamani, R. M. Guedes, F. J. Ferreira, Mech. Time-Depend. Mater. 2016, 20, 45 .

[10] C. R. Siviour, S. M. Walley, W. G. Proud, J. E. Field, Polymer 2005, 46, 12546.

[11] L. H. Zhang, X. H. Yao, S. G. Zang, Q. Han, Mater. Des. 2015, 65, 1181.

[12] G. Spathis, ～E. Kontou, J. Appl. Polym. Sci. 2001, 79, 2534.

[13] J. L. Jordan, J. R. Foley, C. R. Siviour, Mech. Time-Depend. Mater. 2008, 12, 249.

[14] A. D. Mulliken, M. C. Boyce, Int. J. Solids Struct. 2006, 43, 1331.

[15] L. H. Zhang, X. H. Yao, S. G. Zang, Y. B. Gu, Polym. Eng. Sci. 2015, 55, 1864.

[16] J. S. Bergstrom, ～M. C. Boyce, J. Mech. Phys. Solids 1998, 46, 931.

[17] M. Jerabek, Z. Major, R. W. Lang, Polym. Test. 2010, 29, 302. 
[18] P. Li, Z. Wang, Macromol. Mater. Eng. 2016, 301, 577.

[19] S. J. P. Palmer, J. E. Field, J. M. Huntley, Proc. R. Soc. Lond. A 1993, 440, 399.

[20] S. S. Sarva, A. J. Hsieh, Polymer 2009, 50, 3007.

[21] E. M. Arruda, M. C. Boyce, R. Jayachandran, Mech. Mater. 1995, 19, 193.

[22] S. Bahadur, Polym. Eng. Sci. 1973, 13, 266.

[23] V. Dias, C. Odenbreit, O. Hechler, F. Scholzen, T. Ben Zineb, Int. J. Adhes. Adhes. 2014, 48, 194.

[24] A. I. Leonov, Polymer 2005, 46, 5596.

[25] P. Majda, J. Skrodzewicz, Int. J. Adhes. Adhes. 2009, 29, 396.

[26] R. P. Nimmer, Polym. Eng. Sci. 1983, 23, 155.

[27] R. W. Ogden, Proc. R. Soc. Lond. A 1972, 326, 565.

[28] C. Su, Y. J. Wei, L. Anand, Int. J. Plast. 2004, 20, 2063.

[29] T. Beda, J. Polym. Sci. B. 2007, 45, 1713.

[30] M. Danielsson, D. M. Parks, M. C. Boyce, J. Mech. Phys. Solids 2007, 55, 533.

[31] S. Socrate, M. C. Boyce, J. Mech. Phys. Solids 2000, 48, 233.

[32] M. Zehsaz, F. Vakili-Tahami, M. A. Saeimi-Sadigh, J. Strain Anal. Eng. Des. 2015, 50, 4 .

[33] J. S. Bergstrom, M. C. Boyce, Macromolecules 2001, 34, 614.

[34] H. Khajehsaeid, J. Arghavani, R. Naghdabadi, S. Sohrabpour, Int. J. Eng. Sci. 2014, 79, 44.

[35] Z. Wang, P. Li, AIP Adv. 2015, 5, 16.

[36] P. Li, N. V. Nguyen, H. Hao, Mater. Des. 2016, 89, 636.

[37] R. Huang, P. Li, Z. Wang, T. Liu, Adv. Eng. Mater. 2016, 18, 1550. 
[38] B. Brule, J. L. Halary, L. Monnerie, Polymer 2001, 42, 9073.

[39] L. P. Chen, A. F. Yee, E. J. Moskala, Macromolecules 1999, 32, 5944.

[40] D. Rana, V. Sauvant, J. L. Halary, J. Mater. Sci. 2002, 37, 5267.

[41] F. Rietsch, B. Bouette, European Polymer Journal 1990, 26, 1071.

[42] P. Li, Mater. Sci. Eng. A 2015, 622, 114.

[43] W. G. Knauss, J. Zhao, Mech. Time-Depend. Mater. 2007, 11, 199.

[44] J. Richeton, S. Ahzi, L. Daridon, Y. Remond, Polymer 2005, 46, 6035.

[45] J. Richeton, S. Ahzi, K. S. Vecchio, F. C. Jiang, R. R. Adharapurapu, Int. J. Solids Struct. 2006, 43, 2318.

[46] H. Pouriayevali, S. Arabnejad, Y. B. Guo, V. P. W. Shim, Int. J. Impact Eng. 2013, 62, 35 .

[47] E. Ghorbel, Int. J. Plast. 2008, 24, 2032. 


\section{List of Figures}

Fig. 1 (a) The recorded incident, reflected and transmitted wave signals in the SHPB test of a Hysol adhesive at the strain rate of $1000 \mathrm{~s}^{-1}$, and (b) the subsequently calculated stress and strain histories of the adhesive.

Fig. 2 Verification of force equilibrium at the specimen ends in the SHPB experiment (strain rate $1000 \mathrm{~s}^{-1}$ ) of a Hysol adhesive specimen using the parameter $\eta$.

Fig. 3 The representative measured (solid lines) nominal stress versus strain curves of Hysol adhesives at different strain rates, compared to the fitted or predicted curves (dashed lines).

Fig. 4 Recorded deformation and calculated normalised volume of a Hysol specimen at different strain stages under quasi-static compression at the strain rate $0.001 \mathrm{~s}^{-1}$.

Fig. 5 The initial elastic modulus of Hysol adhesives as a function of strain rate.

Fig. 6 The yield strength of Hysol adhesives as a function of strain rate.

Fig. 7 The relation between the normalised true flow stress and the strain rate at various strain stages. Note that the true plastic strain $\varepsilon_{\mathrm{pl}}$ is used. The normalised true flow stress for the $\varepsilon_{\mathrm{pl}}=0.7$ is 1.228 at the rate $0.001 \mathrm{~s}^{-1}$ (outside the figure).

Fig. 8 A Maxwell model to characterise the viscoelastic behaviour of Hysol adhesives.

Fig. 9 Flow chart of regression analysis to determine the material constants in the developed visco-elasto-plastic constitutive model. 
a

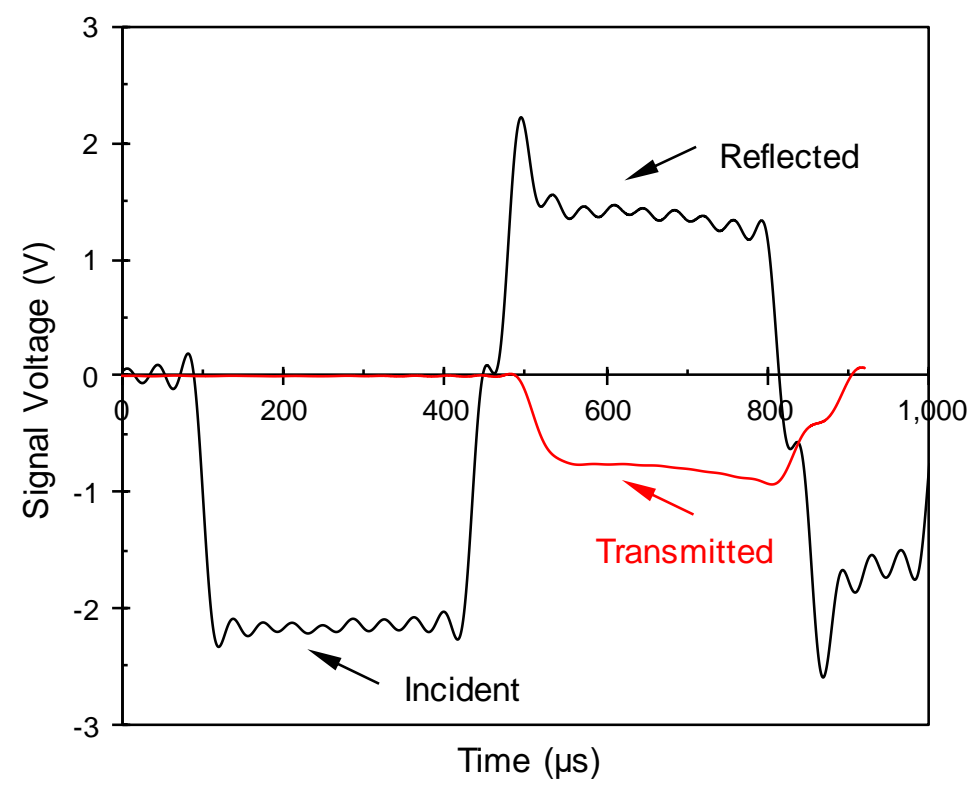

b

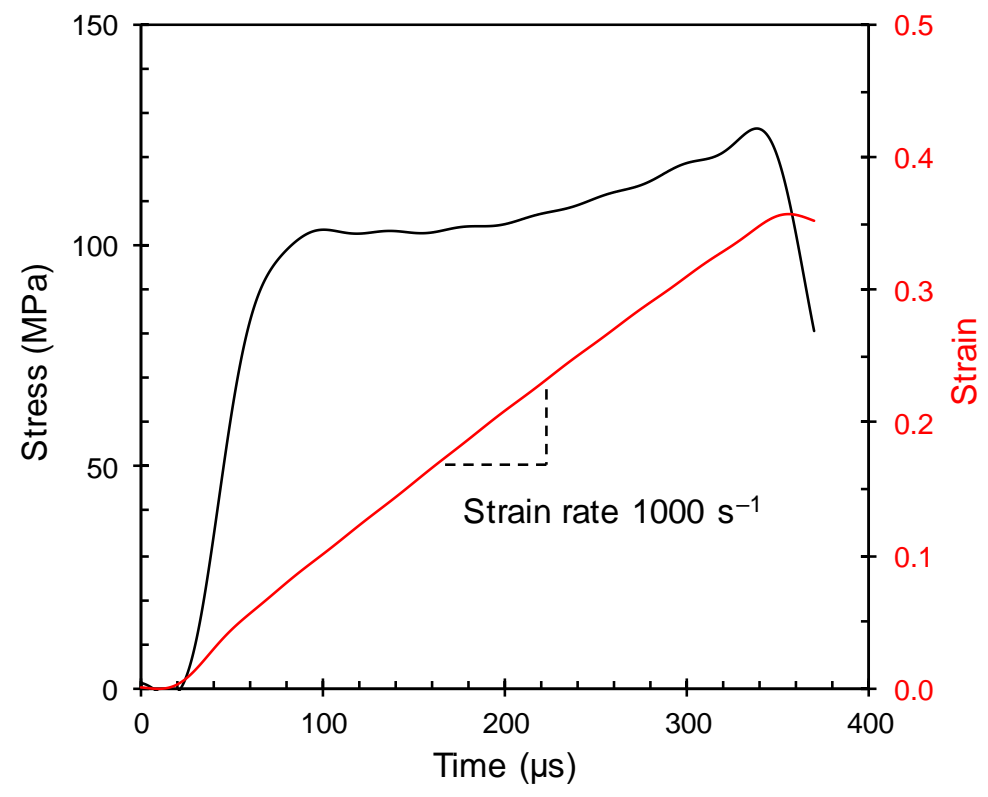

Fig. 1 (a) The recorded incident, reflected and transmitted wave signals in the SHPB test of a Hysol adhesive at the strain rate of $1000 \mathrm{~s}^{-1}$, and (b) the subsequently calculated stress and strain histories of the adhesive. 


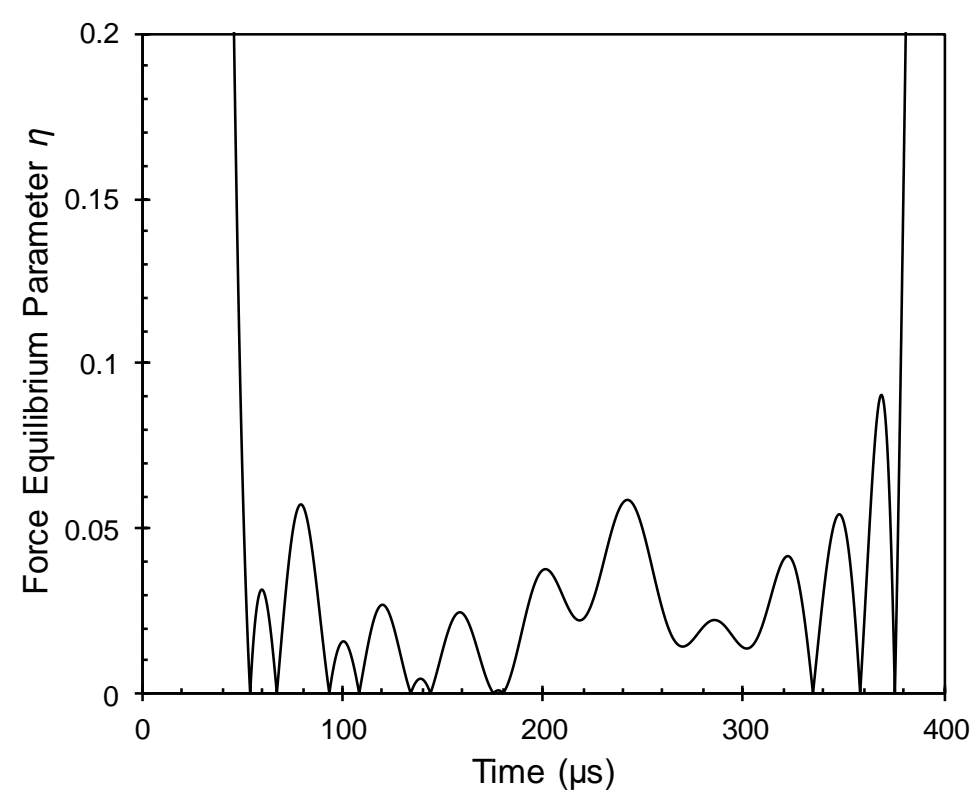

Fig. 2 Verification of force equilibrium at the specimen ends in the SHPB experiment (strain rate $1000 \mathrm{~s}^{-1}$ ) of a Hysol adhesive specimen using the parameter $\eta$. 
a

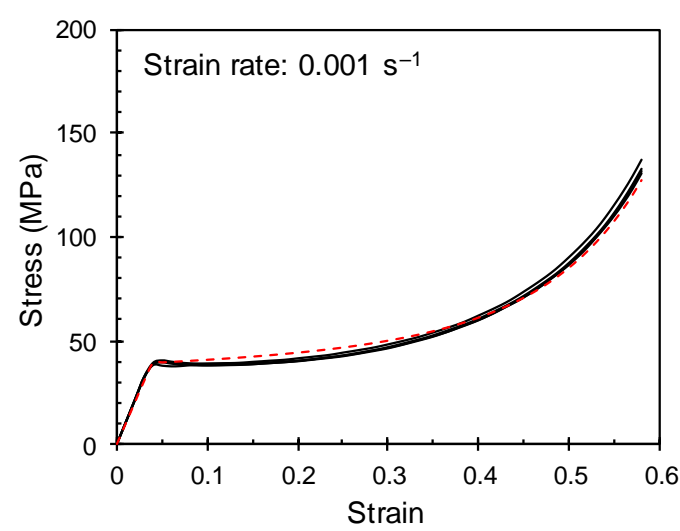

C

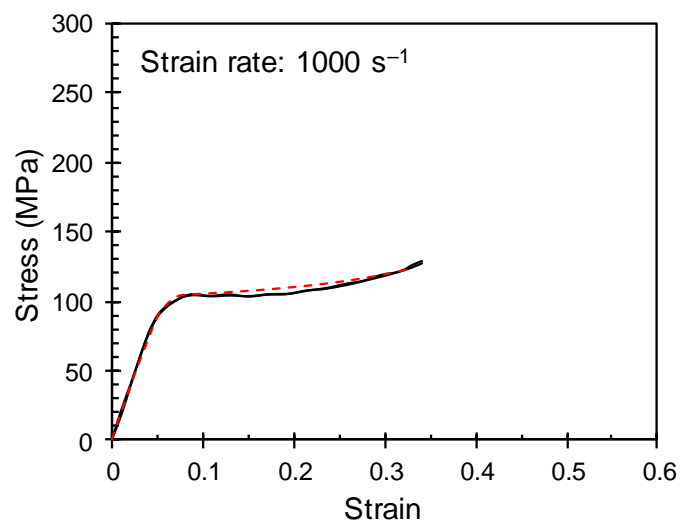

e

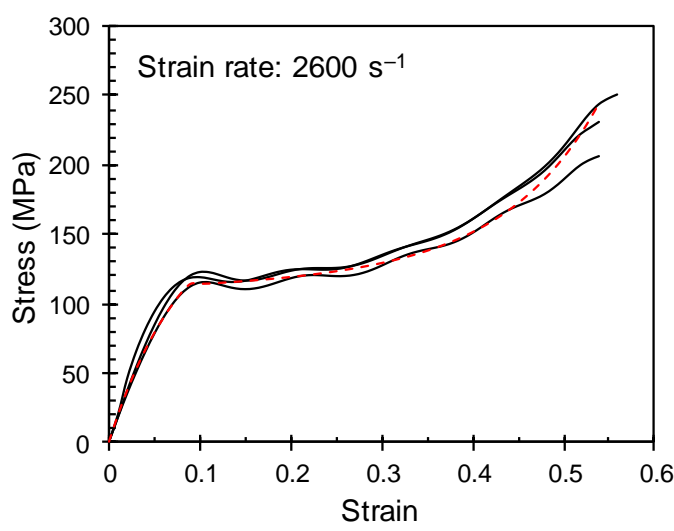

b

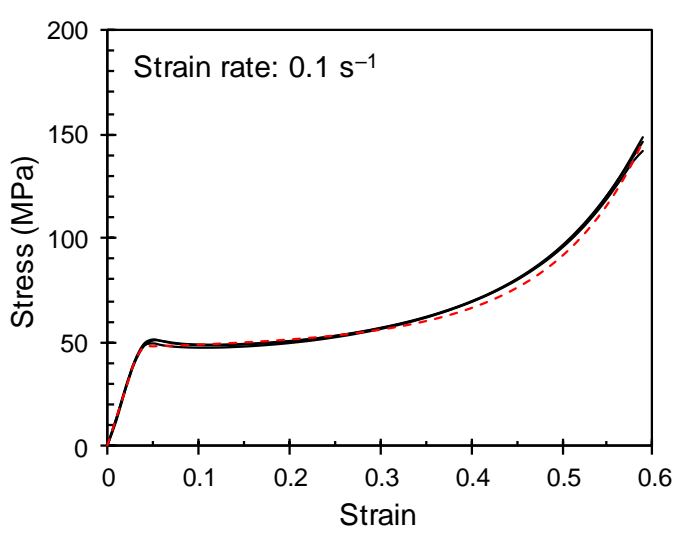

d

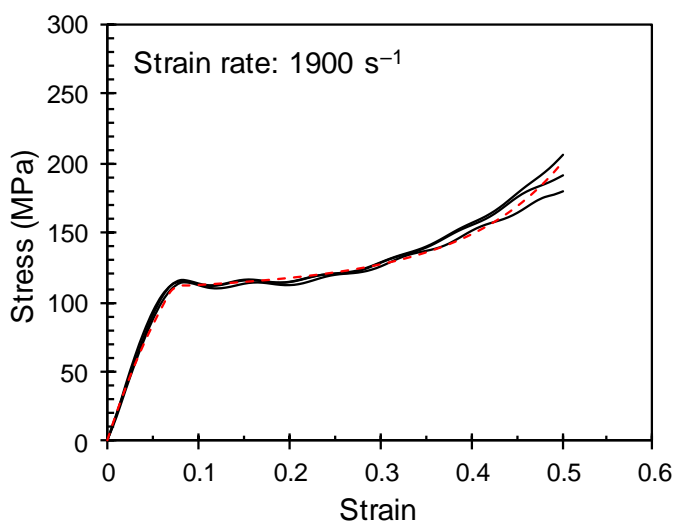

Fig. 3 The representative measured (solid lines) nominal stress versus strain curves of Hysol adhesives at different strain rates, compared to the fitted or predicted curves (dashed lines). 


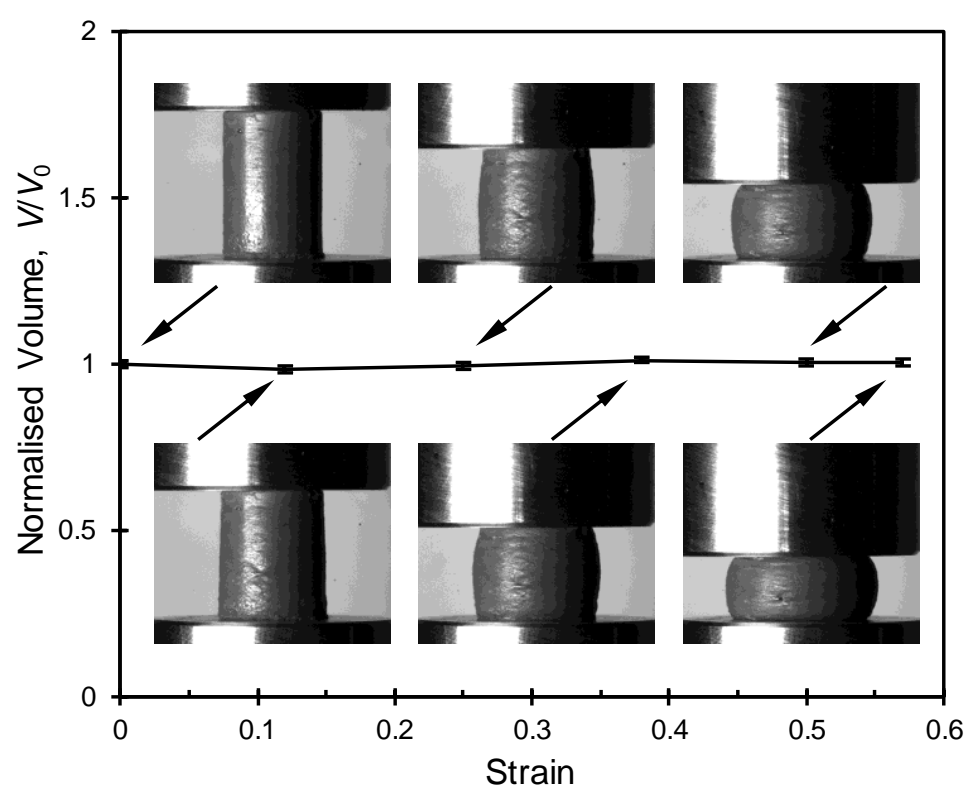

Fig. 4 Recorded deformation and calculated normalised volume of a Hysol specimen at different strain stages under quasi-static compression at the strain rate $0.001 \mathrm{~s}^{-1}$.

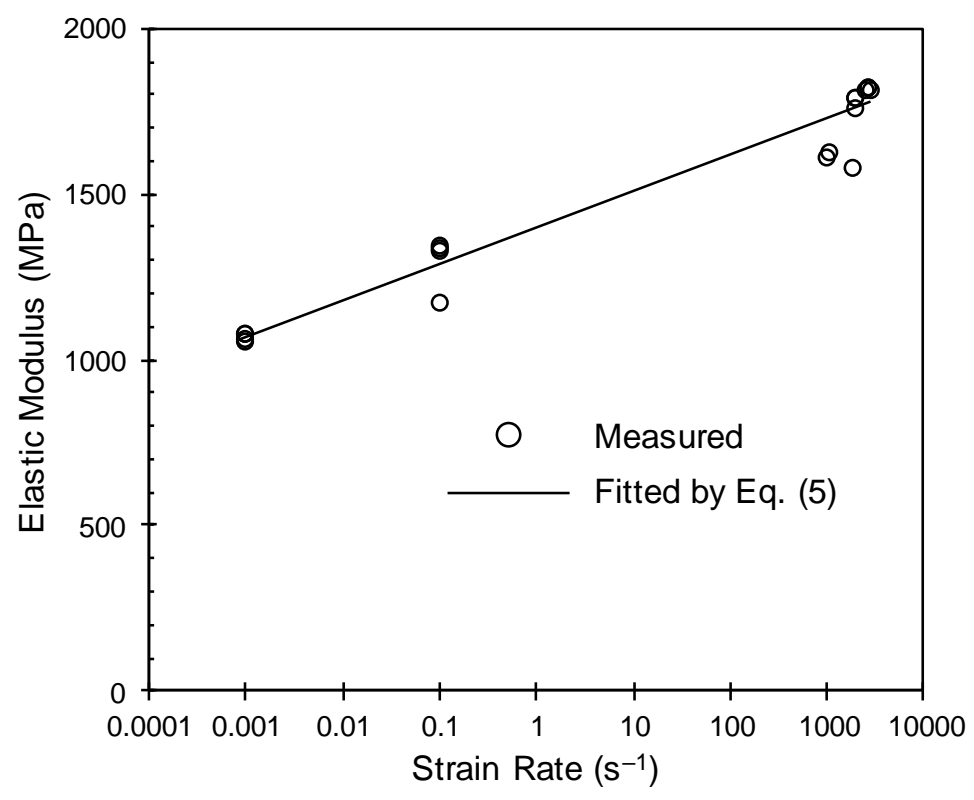

Fig. 5 The initial elastic modulus of Hysol adhesives as a function of strain rate. 


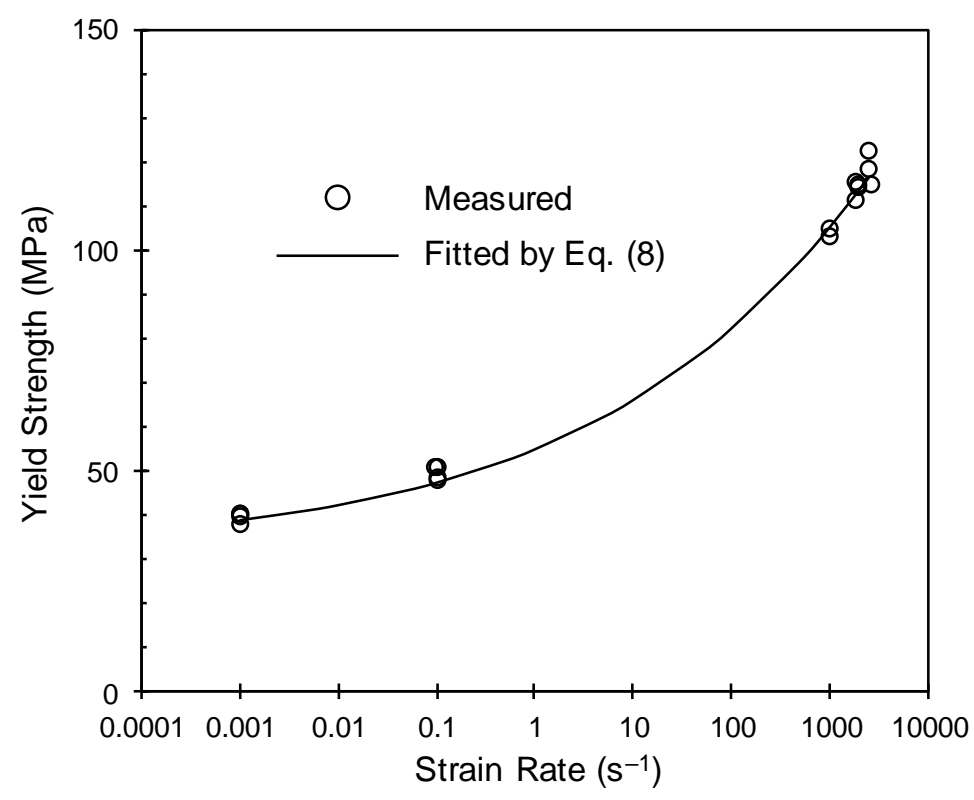

Fig. 6 The yield strength of Hysol adhesives as a function of strain rate.
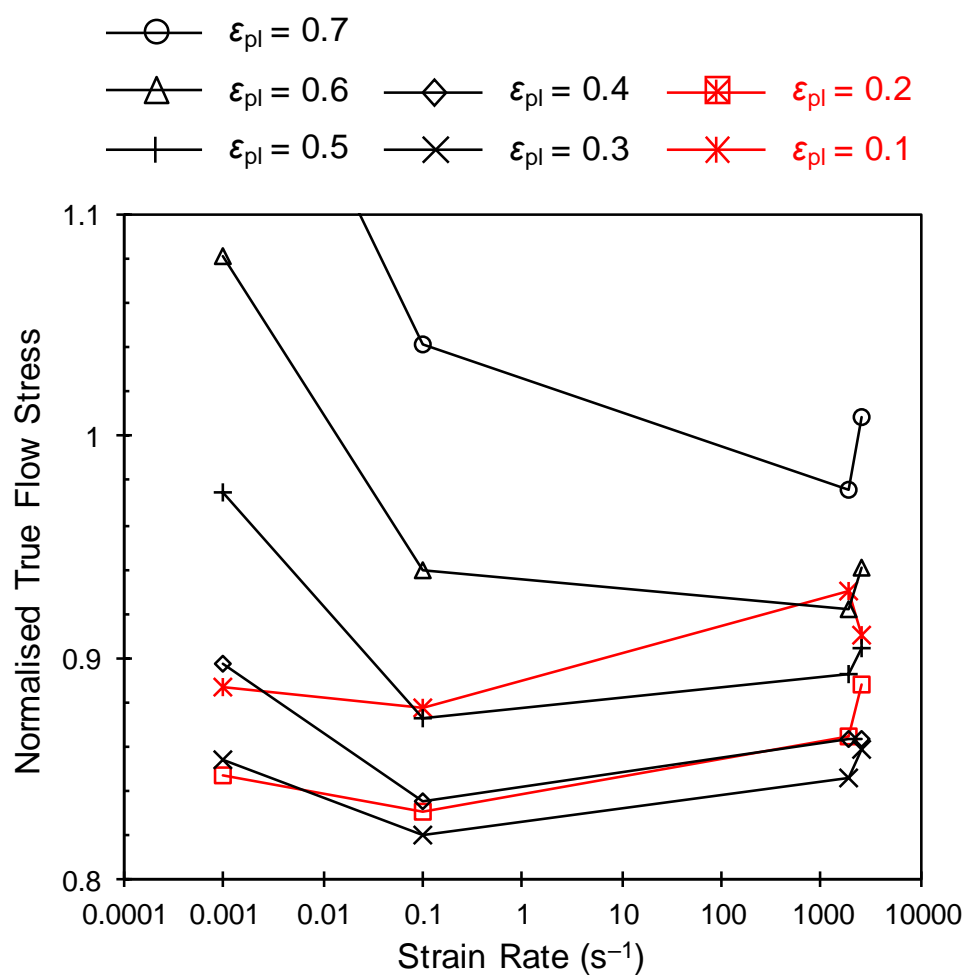

Fig. 7 The relation between the normalised true flow stress and the strain rate at various strain stages. Note that the true plastic strain $\varepsilon_{\mathrm{pl}}$ is used. The normalised true flow stress for the $\varepsilon_{\mathrm{pl}}=0.7$ is 1.228 at the rate $0.001 \mathrm{~s}^{-1}$ (outside the figure). 


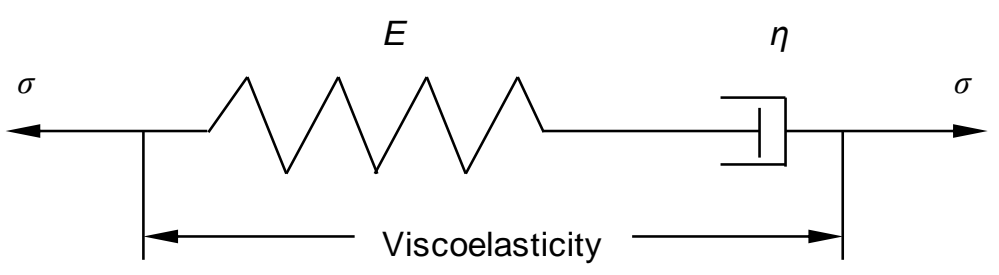

Fig. 8 A Maxwell model to characterise the viscoelastic behaviour of Hysol adhesives.

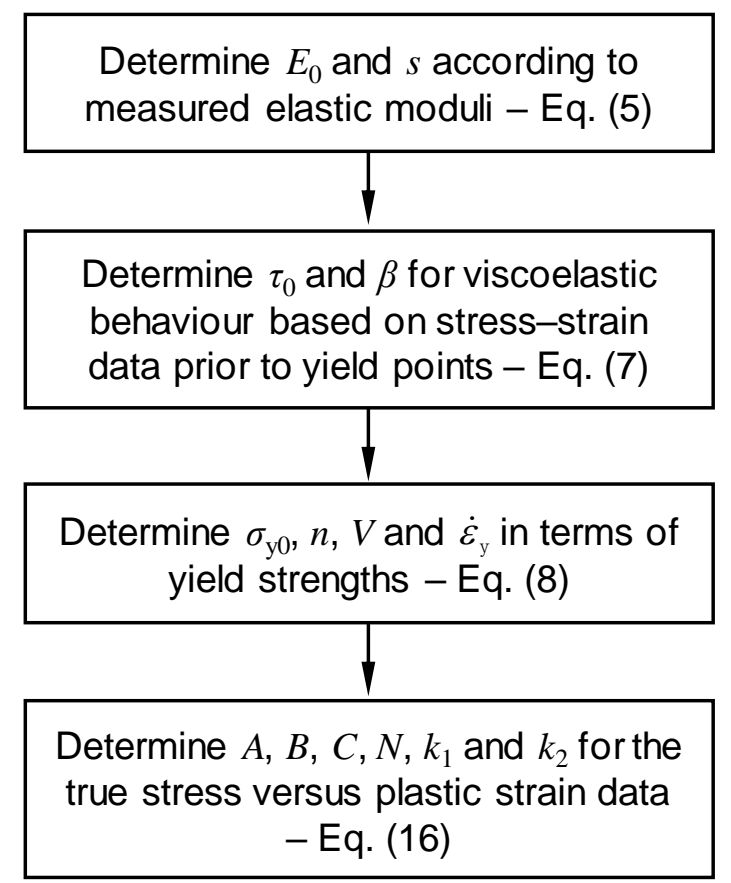

Fig. 9 Flow chart of regression analysis to determine the material constants in the developed visco-elasto-plastic constitutive model. 


\section{List of Tables}

Table 1 Constitutive parameters of the Hysol adhesive.

\begin{tabular}{lc}
\hline Parameter & Value \\
\hline$E_{0}(\mathrm{MPa})$ & 1504 \\
$s$ & 0.0479 \\
$\dot{\varepsilon_{0}}\left(\mathrm{~s}^{-1}\right)$ & 1.0 \\
$\tau_{0}(\mathrm{~s})$ & 243.9 \\
$\beta$ & 2.011 \\
$T_{0}(\mathrm{~K})$ & 298 \\
$k\left(\mathrm{~m}^{2} \mathrm{~kg} \mathrm{~s}^{-2} \mathrm{~K}^{-1}\right)$ & $1.38064852 \times 10^{-23}$ \\
$\sigma_{\mathrm{y} 0}(\mathrm{MPa})$ & 32.22 \\
$n$ & 5.734 \\
$V\left(\mathrm{~m}^{3}\right)$ & $6.354 \times 10^{-29}$ \\
$\dot{\varepsilon_{\mathrm{y}}}\left(\mathrm{s}^{-1}\right)$ & 20530 \\
$A$ & 4.156 \\
$B$ & 0.000228 \\
$C$ & -0.7645 \\
$k_{1}$ & 3.844 \\
$k_{2}$ & 0.1936 \\
\hline & 0.1090 \\
\hline & \\
&
\end{tabular}

